\title{
Amadeus
} International Multidisciplinary Journal IISS 2525-8281

DOI: 10.14295/aimj.v4i8.100

\section{Coleperitoneus Secondary to Acute Alitiasal Cholecystitis: A Case Report}

Whallyson Pinheiro

Mascarenhas ${ }^{1}$

Stenio Santos Moura ${ }^{2}$,

Lucas Benevides Maia ${ }^{3}$

Pedro Hugo Bezerra Maia

Filho $^{4}$,

Mondeyv de Freitas

Pascoal $^{5}$,

Francisco Felippe Araújo

Rolim $^{6}$

\section{Coleperitôneo Secundário à Colecistite Aguda Alitiásica: Relato de Caso}

Abstract: Acute cholecystitis is one of the main causes of medical care in emergencies around the world. It is defined as inflammation of the gallbladder caused mainly by obstruction of the cystic duct by stones. In a small number of patients, the inflammatory process can develop in the absence of stones, receiving the name of alitiásic cholecystitis, having a faster evolution and often evolving with perforation. Acute alitiásic cholecystitis (AAC) corresponds to $15 \%$ of cases of acute cholecystitis and mainly affects patients in severe condition and with comorbidities. Its pathophysiology is still little known, but ischemia and stasis are identified as the main causes. Its high mortality is related to biliary peritonitis due to coleperitoneum. A patient admitted to the emergency department of Hospital Regional do Cariri with acute inflammatory abdomen was diagnosed with coleperitoneum, due to AAC complication, and underwent urgent laparoscopic cholecystectomy, with good clinical evolution in the postoperative period and early return to his activities.

Keywords: acute cholecystitis, coleperitoneum, cholecystectomy

\begin{abstract}
Resumo: A colecistite aguda é uma das principais causas de atendimento médico nas emergências de todo o mundo. É definida como inflamação da vesícula biliar causada, principalmente, pela obstrução do ducto cístico por cálculos. Em uma pequena parcela de pacientes, pode se desenvolver o processo inflamatório na ausência de cálculos, recebendo o nome de colecistite alitiásica, tendo uma evolução mais rápida e frequentemente evolui com perfuração. A colecistite aguda alitiásica (CAA) corresponde a $15 \%$ dos casos de colecistite aguda e acomete principalmente pacientes em estado grave e com comorbidades. Possui fisiopatologia ainda pouco conhecida, porém isquemia e estase são apontadas como as principais causas. Sua elevada mortalidade está relacionada à peritonite biliar por coleperitôneo. Um paciente admitido na emergência do Hospital Regional do Cariri com quadro de abdome agudo inflamatório foi diagnosticado com coleperitôneo, devido complicação da CAA, sendo submetido à colecistectomia videolaparoscópica de urgência, apresentando boa evolução clínica no pósoperatório e retorno precoce as suas atividades.
\end{abstract}

Palavras-chave: colecistite aguda alitiásica, colecistite aguda, coleperitôneo,

\footnotetext{
${ }^{1}$ Residente Cirurgia Geral- Hospital Regional do Cariri, Juazeiro do Norte-CE, Brasil, whallyson10@ hotmail.com;

${ }^{2}$ Residente Cirurgia Geral- Hospital Regional do Cariri, Juazeiro do Norte-CE, Brasil, stenio_moura33@ hotmail.com;

${ }^{3}$ Residente Cirurgia Geral- Hospital Regional do Cariri, Juazeiro do Norte-CE, Brasil, benevides973@ hotmail.com;

${ }^{4}$ Residente Cirurgia Geral- Hospital Regional do Cariri, Juazeiro do Norte-CE, Brasil, pedrohugomaia@ hotmail.com;

${ }^{5}$ Cirurgião do Aparelho Digestivo e Coordenador da Residência de Cirurgia Geral do Hospital Regional do Cariri - HRC. Juazeiro do

Norte, CE - Brasil, mondeyv@gmail.com;
} 
${ }^{6}$ Cirurgião Geral e Professor da Residência de Cirurgia Geral no Hospital Regional do Cariri - HRC. Juazeiro do Norte, CE - Brasil, felippe.rolim1@gmail.com

\section{Introdução}

A colecistite aguda é o processo de inflamação da vesícula biliar causado, principalmente, pela obstrução do ducto cístico por cálculos, impedindo a saída de bile. (Huffman; Schenker, 2010). É apontada como uma das principais causas de atendimento nos serviços médicos de emergência em todo o mundo. Quando esse processo inflamatório agudo acontece na ausência desses cálculos, recebe o nome de colecistite alitiásica. Porém, apesar de não ser tão comum, pode evoluir para um prognóstico desfavorável em pacientes previamente hospitalizados (Siqueira et al.,2012).

A colecistite alitiásica corresponde a $2-15 \%$ dos casos de colecistite aguda, apresentando-se mais frequentemente em pacientes graves, idosos, politraumatizados, sépticos e do sexo feminino, sendo pouco comum em pacientes fora do ambiente hospitalar (Huffman; Schenker, 2010).

Sua etiologia é pouco compreendida, mas o que se sabe é que a estase, a bile espessa, a isquemia e a injúria por reperfusão são tidas como as principais causas. A principal complicação é a perfuração da vesícula biliar, encontrada em 10\% dos casos de colecistite aguda, cuja mortalidade pode chegar a 30\% das CAA, quando resulta em peritonite biliar generalizada por coleperitôneo (Indar; Beckingham, 2002; Williams; Scobie, 1976).

O presente trabalho tem como finalidade relatar um caso de coleperitôneo secundário à colecistite aguda alitiásica em um paciente previamente hígido.

\section{Relato de Caso}

Paciente JDL de 38 anos, masculino, autônomo, previamente hígido e sem comorbidades, foi admitido na emergência do Hospital Regional do Cariri com quadro de dor abdominal de forte intensidade em hipocôndrio direito há 5 dias, com irradiação para hemitórax direito, região occipital e fossa ilíaca direita, associada à anorexia e náusea. Negava febre, vômitos ou constipação.

Ao exame físico, apresentou-se com estado geral regular, anictérico, corado, desidratado, normotenso, normocárdico e eupneico. Ausculta cardíaca e respiratória sem 
alterações. Abdome plano, depressível, com palpação de massa de contornos irregulares em hipocôndrio direito, sinal de Murphy e sinal de Blumberg positivos e ruídos hidroaéreos presentes, sem sinais de peritonite difusa.

Foi iniciado tratamento empírico com ceftriaxona e metronidazol e solicitado exames complementares. Os exames laboratoriais evidenciaram os seguintes resultados: Hemoglobina: 16,8g/dL; leucócitos: 13.700 células $/ \mathrm{mm}^{3}$ sem desvio à esquerda; plaquetas: 265.000/mm³; Fosfatase Alcalina: 74U/L; Gama GT: 63U/LH; Creatinina: 1,1mg/dL; Ureia: 28,5mg/dL; Bilirrubina Total: 0,74mg/dL; Bilirrubina Direta: 0,32mg/dL; Amilase: 109U/L; Lipase: 148U/L.

A ultrassonografia (USG) de abdome evidenciando imagem tubular em fundo cego, com parede espessada e não compressível, situada em fossa ilíaca direita, sugestivo de apendicite aguda, sem líquido livre em cavidade abdominal. Vesícula biliar parcialmente contraída, sem dilatação de vias biliares e sem sinais de colelitíase ou coledocolitíase. Pâncreas parcialmente visualizado devido interposição gasosa.

A tomografia computadorizada (TC) de abdome mostrou vesícula biliar com conteúdo homogêneo, espessamento difuso de paredes, com irregularidade na porção fúndica e comunicação com coleção líquida em parênquima hepático adjacente ao fundo da vesícula, sem colelitíase. Apêndice vermiforme não individualizado, porém, sem borramento de gordura adjacente e pequena quantidade de líquido livre em hipocôndrio e goteira parietocólica direita.

Devido a não melhora dos sintomas com a terapia inicial, o paciente foi submetido à videolaparoscopia de urgência, onde foi evidenciado coleperitôneo com presença de aproximadamente $100 \mathrm{ml}$ de bile espessa em região de hipocôndrio e goteira parietocólica direita; apêndice vermiforme sem sinais de inflamação; vesícula biliar bloqueada por epíplon, intra-hepática, com paredes espessadas, necrosada e perfurada em região de fundo e pequena quantidade de empiema pericolecístico.

Foi realizada a lavagem da cavidade abdominal, colecistectomia a Torek, onde a parede posterior da vesícula é deixada na superfície hepática e cauteriza-se a mucosa remanescente; ligadura do ducto cístico com fio ethibond 2-0 e deixado dreno laminar em leito hepático.

A evolução foi satisfatória, visto que, $\log 0$ no $1^{\circ}$ dia pós-operatório, o paciente relatou melhora da dor, boa aceitação da dieta oral e evacuações presentes, dreno laminar $20 \mathrm{ml}$ de aspecto seroso, afebril. No $2^{\circ}$ dia pós-operatório, já se encontrava assintomático, 
dreno com débito desprezível, com exames mostrando leucócitos de 10.100, sem outras alterações. Recebeu alta hospitalar nesse mesmo dia, com retirada de dreno e orientações para término do antibiótico intradomiciliar com ciprofloxacino e metronidazol.

Retornou à consulta ambulatorial após 10 dias, referindo melhora completa do quadro inicial e retorno às atividades laborais. Resultado do histopatológico foi de processo inflamatório compatível com colecistite aguda.

\section{Discussão}

A colecistite aguda alitiásica (CAA) é um processo inflamatório da vesícula biliar que acomete, principalmente, pacientes graves ou com comorbidades prévias, além de ser responsável por até 15\% dos casos de colecistite aguda (Barie,;Eachempati, 2003; Ganpathi et al., 2007; Indar; Beckingham, 2002).

De etiologia multifatorial, a CAA ainda tem sua fisiopatologia pouco compreendida. No entanto, sabe-se que a baixa perfusão tecidual, muito comum em pacientes graves e em uso de drogas vasoativas, pode levar à necrose e à perfuração da parede da vesícula; a elevada resposta inflamatória, presente em pacientes politraumatizados e no pós-operatório de cirurgias extensas, e a estase biliar causada por quadros de febre, jejum prolongado e desidratação, podendo levar ao espessamento da bile, são apontados como os principais mecanismos fisiopatológicos relacionados à doença. (Awori; Saidi; Kiptoon, 2006; Laurila, 2006; Siqueira et al., 2012).

No caso relatado, o paciente é jovem, hígido e nega patologias prévias, fugindo do padrão estatístico dessa doença, o que dificultaria ainda mais a investigação diagnóstica durante a abordagem inicial na urgência.

O diagnóstico da CAA é feito através de dados clínicos, laboratoriais e de imagem. As manifestações clínicas nem sempre são conclusivas, podendo cursar com dor ou massa palpável em hipocôndrio direito, sinal de Murphy, febre, náusea, vômitos, icterícia, diarreia e rebaixamento do nível de consciência (Fonseca; Dos Santos Silveira; Borghesi, 2015; Williams; Scobie, 1976).

Os exames laboratoriais com leucocitose ou leucopenia, aumento de fosfatase alcalina e elevação dos marcadores de função hepática também são comuns, porém, inespecíficos e com baixo valor diagnóstico (Huffman; Schenker, 2010). Por ser um paciente do sexo masculino, jovem, com dor irradiada para hemitórax direito, sinal de 
Murphy e Blumberg ao exame físico, com sintomas e exames laboratoriais presentes na maioria dos abdomes agudos inflamatórios, o seguimento com exames laboratoriais e radiológicos foi de fundamental importância no caso em questão para descartar outras hipóteses diagnósticas, como a pancreatite aguda, descartada após resultado normais de amilase e lipase.

Nos exames de imagem, a CAA normalmente é identificada através de achados sugestivos de vesícula biliar distendida, com paredes espessadas, líquido pericolecístico, bile espessa, sem cálculos no seu interior (Siqueira et al.,2012). Entretanto, a ultrassonografia de abdome inicial evidenciou sinais sugestivos de apendicite aguda, sem líquido livre em cavidade, com vesícula contraída e sem sinais de inflamação.

O USG abdominal descreveu interposição gasosa de alças intestinais e esses gases intraluminais podem refletir e produzir uma imagem equivalente à atresia de vesícula. (Araújo et al, 2007; Bouras et al, 2005; Fonseca; Dos Santos Silveira; Borghesi, 2015). Pelo fato deste ser um exame operador dependente, optou-se pela solicitação da tomografia de abdome para auxiliar no diagnóstico e excluir outros focos de infecção. A TC sugeriu colecistite complicada com perfuração, provável abscesso hepático e coleperitôneo.

A mortalidade dos pacientes com CAA é de 30\%, independente da terapia estabelecida. Isso é explicado pela relação proporcional com a gravidade do quadro clínico prévio do paciente e com a rápida evolução para necrose e perfuração da parede da vesícula.

Apesar da distorção anatômica causada pelo processo inflamatório da colecistite aguda perfurada, foi optado pela realização de colecistectomia laparoscópica, por esta causar menor dano celular, menor reação inflamatória e menor tempo de internação hospitalar que a colecistectomia aberta (Huffman; Schenker, 2010).

\section{Conclusões}

Este relato ilustra um caso de coleperitôneo secundário à $\mathrm{CAA}$, em paciente previamente hígido, que teve um desfecho favorável após realização de colecistectomia laparoscópica de urgência, mostrando que, além de auxiliar no diagnóstico, a laparoscopia também pode atuar de forma terapêutica, mesmo em casos complexos, diminuindo as complicações relacionadas à resposta inflamatória causada pela cirurgia aberta. 
Apesar de a mortalidade ser elevada, ela está diretamente relacionada ao quadro clínico inicial do paciente, mostrando que o fato de ser previamente hígido favoreceu o retorno precoce as suas atividades.

\section{Referências}

ARAÚJO, D. B. et al. (2007). Colecistite enfisematosa. RBM Rev Bras Med, v. 63, n. 3, p. 117-9, 2007.

AWORI, K. O.; SAIDI, Saidi H.; KIPTOON, D. K. (2006). Acute acalculous cholecystitis in an outpatient Setting. East and Central African Journal of Surgery, v. 11, n. 2, p. 48-53.

BARIE, Philip S.; EACHEMPATI, Soumitra R. (2003). Acute acalculous cholecystitis. Current gastroenterology reports, v. 5, n. 4, p. 302-309.

BOURAS, George et al. (2005). A case of emphysematous cholecystitis managed by laparoscopic surgery. JSLS: Journal of the Society of Laparoendoscopic Surgeons, v. 9, n. 4, p. 478.

FONSECA, Maria Silian Mandu; DOS SANTOS SILVEIRA, Jaqueline; BORGHESI, Ronaldo Antonio.(2015). Colecistite aguda enfisematosa: um relato de caso. Revista da Faculdade de Ciências Médicas de Sorocaba, v. 17, n. 4, p. 236-239, 2015.

GANPATHI, Iyer Shridhar et al. (2007). Acute acalculous cholecystitis: challenging the myths. HPB: The Official Journal of the International Hepato Pancreato Biliary Association, v. 9, n. 2, p. 131.

HUFFMAN, Jason L.; SCHENKER, Steven. (2010). Acute acalculous cholecystitis: a review. Clinical Gastroenterology and Hepatology, v. 8, n. 1, p. 15-22.

INDAR, Adrian A.; BECKINGHAM, Ian J. (2002). Acute cholecystitis. Bmj, v. 325, n. 7365, p. 639-643.

LAURILA, Jouko. Surgically treated acute acalculous cholecystitis in critically ill patients. University of Oulu, 2006.

SIQUEIRA, Valdirene Silva et al. (2012). Colecistite alitiásica aguda: revisão de literatura. Rev Med Minas Gerais, v. 22, n. Supl 5, p. S59-S62.

WILLIAMS, N. F.; SCOBIE, T. K. (1976). Perforation of the gallbladder: analysis of 19 cases. Canadian Medical Association Journal, v. 115, n. 12, p. 1223.

\section{How to cite this article (APA format):}

Mascarenhas, Whallyson Pinheiro; Moura, Stenio Santos; Maia, Lucas Benevides; Maia Filho, Pedro Hugo Bezerra; Pascoal, Mondeyv de Freitas; Rolim, Francisco Felippe Araújo (2020). Coleperitoneus Secondary to Acute Alitiasal Cholecystitis: A Case Report. Am. In. Mult. J., February to May. (8) 5, 19-24.

Received: 01/14/2020;

Accepted: 01/17/2020. 